\title{
BOND INVARIANCE OF $G$-RINGS AND LOCALIZATION
}

\author{
ROBERT B. WARFIELD, JR.
}

(Communicated by Maurice Auslander)

\begin{abstract}
It is proved that if $R$ and $S$ are prime Noetherian rings and there exists an $(R, S)$-bimodule that is finitely generated and torsionfree on each side, then the intersection of the nonzero prime ideals of $R$ is nonzero if and only if the same holds for the corresponding intersection in $S$. Consequently, if the right primitive ideais in a given Noetherian ring are precisely the locally closed prime ideals, then the same equivalence holds true for any finite extension ring. Another consequence of the methods used here is the following answer to a question of Braun: If the intersection of the prime ideals in a clique in a Noetherian PI ring is a prime ideal $Q$, then $Q$ is localizable.
\end{abstract}

\section{INTRODUCTION}

This paper is concerned with the interaction between the prime spectra of Noetherian rings connected by torsionfree bimodules, with applications given to ring extensions, and to localization theory. To start, recall that a prime ring is a G-ring provided that the intersection of all of the nonzero prime ideals is itself nonzero. A $G$-ideal of a ring $R$ is a prime ideal $P$ such that $R / P$ is a $G$-ring. (A prime $P$ in $R$ is a $G$-ideal if and only if $P$ is locally closed in $\operatorname{Spec}(R)$.) If $R$ and $S$ are prime Noetherian rings, and there exists a nonzero $(R, S)$-bimodule ${ }_{R} B_{S}$ that is finitely generated and torsionfree both as a left $R$-module and as a right $S$-module, then we say that $R$ is bonded to $S$ and that $B$ is a bond from $R$ to $S$. We prove: If $R$ and $S$ are bonded prime Noetherian rings then $R$ is a G-ring if and only if $S$ is a G-ring.

There is an immediate consequence to the above result. Let $V$ be a Noetherian ring containing a Noetherian subring $U$ such that $V$ is finitely generated as both a left and a right $U$-module. We obtain the following corollary: If in $U$ an arbitrary prime is right primitive exactly when it is a G-ideal, then the same holds for an arbitrary prime of $V$. This characterization of primitivity holds in

Received by the editors October 30, 1989.

1980 Mathematics Subject Classification (1985 Revision). Primary 16A33, 16A38, 16 A66.

Key words and phrases. Noetherian ring, Noetherian bimodule, bond, prime ideal, primitive ideal, $G$-ring, locally closed, ring extension, clique, localization, PI ring.

This research was partially supported by a National Science Foundation grant.

The submitted version of this paper was prepared from the author's original draft by $K$. $R$. Goodearl and E. S. Letzter. 
particular when $U$ is the enveloping algebra of a finite-dimensional Lie algebra over a field of characteristic zero (see, for example, [2] or [6]).

Our second interest is in a question of A. Braun: If the intersection of the primes in a clique in a Noetherian PI ring is a prime $Q$, must $Q$ be localizable? (We refer the reader to [1,3, or 5] for definitions of unexplained terms.) Using a modification of the above result on bond invariance of the $G$-ring property, we obtain an affirmative answer. We also consider certain generalizations of this question.

\section{I. $G$-RINGS}

We begin by stating our main result on $G$-rings, which follows immediately from Theorem 3 .

Theorem 1. Let $R$ and $S$ be Noetherian prime rings and ${ }_{R} B_{S}$ a bond between them. Then $S$ is a $G$-ring if and only if $R$ is a G-ring.

Corollary 2. Let $U$ be a Noetherian subring of a ring $V$ such that $V$ is finitely generated as both a left and a right $U$-module. Suppose that one of the following properties holds:

(a) All right primitive ideals of $U$ are $G$-ideals.

(b) All $G$-ideals of $U$ are right primitive.

Then the same property holds for $V$.

Remark. Corollary 2 extends results of Letzter [4, Theorems 2.3, 2.4] on finite free extensions.

Proof of Corollary 2. Let $P$ be a prime ideal of $V$. Using [4, Lemma 1.1] or [1, Proposition 7.7], we see that if $\left\{Q_{1}, \ldots, Q_{m}\right\}$ are the primes of $U$ minimal over $P \cap U$, then there are bonds between $U / Q_{i}$ and $V / P$, and also between $V / P$ and $U / Q_{i}$. Since right primitivity transfers from left to right across bonds [4, Lemma 1.3; 1, Theorem 7.16], the result follows from Theorem 1.

We actually prove a slightly generalized form of Theorem 1 which has further applications in localization theory.

If ${ }_{R} B_{S}$ is a bond between Noetherian prime rings $R$ and $S$, and $P$ and $Q$ are primes of $R$ and $S$ respectively, then we say there is a $B$-bond from $R / P$ to $S / Q$ if there are sub-bimodules $C$ and $D$ of $B$ with $C \geq D$ such that $C / D$ is a bond from $R / P$ to $S / Q$.

Theorem 3. Let $R$ and $S$ be Noetherian prime rings and ${ }_{R} B_{S}$ a bond between them. Let $\Delta$ be a collection of nonzero prime ideals of $R$ and $\Delta^{*}$ the collection of those prime ideals $Q$ of $S$ such that for some $P \in \Delta$ there is a $B$-bond from $R / P$ to $S / Q$. Suppose that $\cap\{P: P \in \Delta\}=0$. Then $\cap\left\{Q: Q \in \Delta^{*}\right\}=0$.

Remark. It is a consequence of [1, Proposition 7.4$]$ that the primes in $\Delta^{*}$ are nonzero.

Proof. Suppose to the contrary that $\bigcap\left\{Q: Q \in \Delta^{*}\right\}=J \neq 0$. Since $B / B J$ is torsion as a right $S$-module, there is a nonzero ideal $K$ of $R$ with $K B \leq B J$ 
[1, Proposition 7.4]. Let $T$ be the trace ideal of $B$ in $R$, that is, the ideal generated by $f(B)$ for all $f \in \operatorname{Hom}\left({ }_{R} B,{ }_{R} R\right)$. Since $R$ is prime, $T \cap K \neq 0$. Hence for some $P \in \Delta$ we have $P \nsupseteq T \cap K$.

We define $M$ so that $B / M$ is the largest possible factor of ${ }_{R} B$ which is a torsionfree left $(R / P)$-module. That is, $M / P B$ is the torsion submodule of ${ }_{R / P}(B / P B)$. Since $P \geq T$, there exist nonzero $R$-module homomorphisms ${ }_{R} B \rightarrow R / P$, so $B \neq M$ and $B / M$ is a nonzero bimodule that is torsionfree as a left $(R / P)$-module. Let

$$
0=B_{0} / M<B_{1} / M<\cdots<B_{n-1} / M<B_{n} / M=B / M
$$

be a right affiliated series for $B / M$, with right affiliated primes $Q_{1}, \ldots, Q_{n}$. By [1, Propositions 7.5, 7.7] each $B_{k} / B_{k-1}$ is a bond between $R / P$ and $S / Q_{k}$, so $Q_{k} \in \Delta^{*}$ for all $k, 1 \leq k \leq n$. Let $Q=Q_{n}$ and $C=B_{n-1}$. Then

$$
C \geq B Q \geq B J \geq K B \text {, }
$$

and so the nonzero torsionfree $(R / P)$-module $B / C$ is a homomorphic image of $B /(P+K) B$. However, $P \geq K$ so $B /(P+K) B$ is a torsion left $(R / P)$-module. This contradiction proves the theorem.

\section{Application to localization}

The original purpose of this section was to answer the following question raised by A. Braun: If $C$ is a clique of primes in a Noetherian PI ring such that $Q=\bigcap\{P: P \in C\}$ is prime, is $Q$ necessarily localizable? We put an affirmative answer in a more general context.

The natural class of rings in which to discuss localization is the class of Noetherian rings with the second layer condition, and we refer to [3] or [1] for a discussion of these rings. If $P$ and $Q$ are primes in a Noetherian ring $R$ then there is a link from $P$ to $Q$, written $P \leadsto Q$, if there is an ideal $A$ such that $P \cap Q>A \geq P Q$ and $(P \cap Q) / A$ is a bond between $R / P$ and $R / Q$. These links can be used to turn $\operatorname{Spec}(R)$ into a graph and the connected components of this graph are the cliques of primes in $R$. A major point of the theory is that it is necessary to localize at an entire clique of primes rather than at individual prime ideals. If a clique of primes in a Noetherian ring with the second layer condition is finite then it is localizable (see e.g. [3, Theorem 7.3.1; 5, Theorem 4.3.16; or 1, Theorem 12.21]), but the general situation is less clear.

In this section we consider a (presumably infinite) clique $C$ of primes and ask about the intersection of the ideals in $C$. This semiprime ideal is not in general localizable but in a certain sense part of it is.

Theorem 4. Let $R$ be a Noetherian ring with the second layer condition, $C$ a clique of primes in $R$, and $N=\bigcap\{P: P \in C\}$. Let the primes minimal over $N$ be $Q_{1}, \ldots, Q_{n}$. Let $\alpha_{i}=\mathrm{Cl} \cdot \mathrm{K} \cdot \operatorname{dim}\left(R / Q_{i}\right)$ and suppose that $\alpha_{1}=\alpha_{2}=\cdots=$ $\alpha_{k}$ while $\alpha_{m}<\alpha_{1}$ for $k<m \leq n$. Let $N^{\prime}=Q_{1} \cap \cdots \cap Q_{k}$. Then $N^{\prime}$ is a localizable semiprime ideal. 
Corollary 5. Let $R$ be a Noetherian ring satisfying the second layer condition and $C$ a clique in $\operatorname{Spec}(R)$ such that $Q=\bigcap\{P: P \in C\}$ is a prime ideal. Then $Q$ is localizable.

Proof of Theorem 4. If $N_{i}=\bigcap\left\{P \in C \mid P \geq Q_{i}\right\}$ for each $i$, then

$$
N_{1} N_{2} \cdots N_{n} \leq N_{1} \cap \cdots \cap N_{n}=N=Q_{1} \cap \cdots \cap Q_{n},
$$

and so for each $i$ there exists $j(i)$, where $1 \leq j(i) \leq n$, such that $N_{j(i)} \leq Q_{i}$. Since $Q_{j(i)} \leq N_{j(i)}$, and since the $Q_{i}$ are incomparable, $j(i)=i$ and $Q_{i}=N_{i}$. Thus $Q_{i}$ is an intersection of some primes from the clique $C$.

Recall from [3, Theorem 8.2.4] that if there is an $R$-bond from $R / P$ to $R / P^{\prime}$ with $P \in C$, then $P^{\prime} \in C$ also.

Consider a link $Q_{i} \rightsquigarrow Q^{\prime}$ where $1 \leq i \leq k$. From Theorem 3 and what we have just observed, it follows that $Q^{\prime}$ is an intersection of primes from $C$. Hence $Q^{\prime} \geq N$. Since $1 \leq i \leq k$ we have

$$
\text { Cl.K.dim }\left(R / Q^{\prime}\right)=\text { Cl.K.dim }\left(R / Q_{i}\right)=\alpha_{1}
$$

(e.g. [3, Theorem 8.2.8] or [1, Corollary 12.5]), and so $Q^{\prime}$ is necessarily minimal over $N$. Hence $Q^{\prime}=Q_{j}$ for some $j$, where $1 \leq j \leq k$. Similarly, any prime linked to one of $Q_{1}, \ldots, Q_{k}$ must come from this list. Therefore the set $\left\{Q_{1}, \ldots, Q_{k}\right\}$ is a finite clique and hence localizable.

In the inhomogeneous case Theorem 4 fails, as follows.

Example 6. A Noetherian PI ring $R$ with a clique $C$ such that the intersection of the primes in $C$ is not localizable.

Start with a Noetherian PI algebra $S$ over a field $k$ containing an infinite clique $\left\{I_{m}: m \in \mathbb{Z}\right\}$ such that $S / I_{0} \cong k$ and the intersection $I=\bigcap I_{m}$ is prime; then choose a Noetherian PI algebra $T$ over $k$ containing a two-element clique $\left\{J_{0}, J_{1}\right\}$ such that $T / J_{0} \cong k$. (See Remark 7.) There are $k$-algebra homomorphisms $\phi_{1}: S \rightarrow k$ and $\phi_{2}: T \rightarrow k$ with $\operatorname{ker}\left(\phi_{1}\right)=I_{0}$ and $\operatorname{ker}\left(\phi_{2}\right)=$ $J_{0}$. Now set

$$
R=\left\{(s, t) \in S \times T: \phi_{1}(s)=\phi_{2}(t)\right\},
$$

and let $\pi_{1}: R \rightarrow S$ and $\pi_{2}: R \rightarrow T$ be the canonical projections. Since these projections are surjective, the ideals $P_{m}=\pi_{1}^{-1}\left(I_{m}\right)$ and $Q_{n}=\pi_{2}^{-1}\left(J_{n}\right)$ are primes of $R$. Links lift from $S$ back to $R$, and so the primes $P_{m}$ are all in the same clique; likewise $Q_{0}$ and $Q_{1}$ are in the same clique. Moreover,

$$
P_{0}=I_{0} \times J_{0}=Q_{0} .
$$

Thus the set $C=\left\{P_{m}: m \in \mathbb{Z}\right\} \cup\left\{Q_{1}\right\}$ is contained in a single clique of $R$.

To see that $C$ is link-closed, consider a link $P \rightsquigarrow Q$ via a bond $(P \cap Q) / A$, where $P$ and $Q$ are primes of $R$ and one of them lies in $C$. Let $K_{i}=\operatorname{ker}\left(\pi_{i}\right)$ for $i=1,2$. 
Now if $P \cap Q \cap K_{1} \leq A$, then $(P \cap Q) / A$ is annihilated on both sides by $K_{1}$ and so $K_{1} \leq P \cap Q$. Then $K_{1} \leq A$. Thus

$$
(P \cap Q) / A \cong\left(\pi_{1}(P) \cap \pi_{1}(Q)\right) / \pi_{1}(A),
$$

giving us a link $\pi_{1}(P) \rightsquigarrow \pi_{1}(Q)$ in $S$. It follows that $\pi_{1}(P)$ and $\pi_{1}(Q)$ must both be of the form $I_{m}$, and hence $P$ and $Q$ both lie in $C$. On the other hand, if $P \cap Q \cap K_{1} \not \subset A$, then $\left(P \cap Q \cap K_{1}\right) /\left(A \cap K_{1}\right)$ is isomorphic to a nonzero sub-bimodule of $(P \cap Q) / A$. In this case, since $K_{1} K_{2}=K_{2} K_{1}=0$ we get $K_{2} \leq P \cap Q$, and as above we infer that $\pi_{2}(P) \rightsquigarrow \pi_{2}(Q)$ and again $P, Q \in C$. Therefore $C$ is indeed link-closed, and hence it is a clique in $\operatorname{Spec}(R)$.

If $N$ is the intersection of the primes in $C$, then $N=I \times\left(J_{0} \cap J_{1}\right)=$ $\left(I \times J_{0}\right) \cap Q_{1}$, and the primes minimal over $N$ are $I \times J_{0}$ and $Q_{1}$. Since the clique of $Q_{1}$ contains $Q_{0}$, which is not minimal over $N$, we conclude that $N$ is not localizable.

Remark 7. To give some explicit rings $S$ and $T$ satisfying the conditions assumed at the beginning of Example 6, let $k[x]$ be a polynomial ring over a field $k$ of characteristic zero, and let $\alpha$ be the $k$-algebra automorphism of $k[x]$ sending $x$ to $x+1$. If $S$ is the subring of $M_{2}(k[x])$ consisting of matrices of the form $\left(\begin{array}{cc}a & b \\ 0 & \alpha(a)\end{array}\right)$, then the maximal ideals

$$
I_{m}=\left\{\left(\begin{array}{cc}
a & b \\
0 & \alpha(a)
\end{array}\right): a \in(x+m) k[x] \text { and } b \in k[x]\right\}
$$

(for $m \in \mathbb{Z}$ ) form an infinite clique in $S$, with $\cap I_{m}=\left(\begin{array}{cc}0 & k[x] \\ 0 & 0\end{array}\right)$ and $S / I_{m} \cong k$. If $T=\left(\begin{array}{cc}k[x] & k[x] \\ x k[x] & k[x]\end{array}\right)$, then the maximal ideals

$$
J_{0}=\left(\begin{array}{cc}
k[x] & k[x] \\
x k[x] & x k[x]
\end{array}\right) \quad \text { and } J_{1}=\left(\begin{array}{cc}
x k[x] & k[x] \\
x k[x] & k[x]
\end{array}\right)
$$

form a two-element clique in $T$, with $T / J_{n} \cong k$.

In this case the ring $R$ constructed above is a subring of $M_{2}(k[x]) \times M_{2}(k[x])$, and so it can be viewed as a block diagonal subring of $M_{4}(k[x])$; note that $M_{4}(k[x])$ is finitely generated as a right and a left $R$-module. Thus the ring

$$
R^{\dagger}=R+y M_{4}(k[x, y]) \subseteq M_{4}(k[x, y])
$$

is a prime Noetherian PI ring with a clique $C^{\dagger}$ whose intersection is a nonlocalizable semiprime ideal.

\section{REFERENCES}

1. K. R. Goodearl and R. B. Warfield, Jr., An introduction to noncommutative Noetherian rings, London Math. Soc. Student Text Ser., vol. 16, Cambridge Univ. Press, Cambridge, 1989.

2. R. S. Irving and L. W. Small, On the characterisation of primitive ideals in enveloping algebras, Math. Z. 173 (1980), 217-221. 
3. A. V. Jategaonkar, Localization in Noetherian rings, London Math. Soc. Lecture Note Ser., vol. 98, Cambridge Univ. Press, Cambridge, 1986.

4. E. S. Letzter, Primitive ideals in finite extensions of Noetherian rings, J. London Math. Soc. (2) 39 (1989), 427-435.

5. J. C. McConnell and J. C. Robson, Noncommutative Noetherian rings, Wiley-Interscience, New York, 1987.

6. C. Moeglin, Idéaux primitifs des algèbres enveloppantes, J. Math. Pures Appl. 59 (1980), 265-336.

Department of Mathematics, University of Washington, Seattle, Washington 98195 